\title{
The safety and efficacy of MPR-CTU combined with precise intraoperative ultrasonography guided flexible ureteroscope in the treatment of renal cystic disease
}

\author{
RONGJIANG WANG, NING WANG, JIANER TANG, YU CHEN and JIANGUO GAO \\ Department of Urology, The First Affiliated Hospital of \\ Huzhou Teacher's College, Huzhou, Zhejiang 313000, P.R. China
}

Received May 29, 2017; Accepted October 4, 2017

DOI: $10.3892 /$ etm.2017.5379

\begin{abstract}
The safety and efficacy of multi-planar reconstruction (MPR) image post-processing technique-computed tomography (CT) urography (CTU) combined with precise intraoperative ultrasonography guided flexible ureteroscope in renal cyst incision and drainage in the treatment of cystic diseases of kidney were evaluated. A total of 68 patients were randomly divided into control and observation group $(n=34)$. All the patients were treated with renal cyst incision and drainage under flexible ureteroscope. The control group was under ultrasound guidance. The observation group was combined with MPR-CTU, the safety and efficacy was compared. There was no significant difference between the two groups in the success rate and the time of cyst treatment $(\mathrm{P}>0.05)$. The incidence of intraoperative and postoperative complications of the observation group was significantly lower than that of the control group. After 1 month follow-up, the total effective rate of the observation group was significantly higher than that of the control group, the difference was statistically significant $(\mathrm{P}<0.05)$. Conclusion MPR-CTU technique combined with intraoperative ultrasonography to guide cyst incision and drainage under flexible ureteroscope for renal cystic disease has a high safety and efficacy, and it is worthy of clinical application.
\end{abstract}

\section{Introduction}

Cystic diseases of kidney (CDK) refers to a variety of complex diseases with the same morphological features of renal cysts, including simple renal cysts, multiple/multilocular renal cysts, parapelvic cyst, medullary sponge kidney, calyceal diverticulum and other non-hereditary diseases, and autosomal dominant hereditary polycystic kidney disease,

Correspondence to: Dr Rongjiang Wang, Department of Urology, The First Affiliated Hospital of Huzhou Teacher's College, 158 Guangchanghou Road, Huzhou, Zhejiang 313000, P.R. China E-mail: hzyymnwk@163.com

Key words: multi-planar reconstruction, CT urography, flexible ureteroscope, renal cyst incision and drainage multiple malformations with renal cyst and other genetic diseases (1). Clinical treatment includes puncture drainage and sclerotherapy injection, laparoscopic surgery, percutaneous nephrolithotomy, ureteroscopy and open surgery $(1,2)$.

Renal cyst incision and drainage under ureteroscope is developed on the basis of the wide use of ureteroscope in examination and lithotripsy, with the advantage of passing the natural cavity path and is minimally invasive (1). Intraoperative ultrasound can provide precise real-time positioning for cysts, especially thick-walled cysts, guiding the incision direction of ureteroscope (2). The renal cyst adjacent to the calyx on the transverse position of two-dimensional CT cannot be fully positioned, which reduces the success rate of fenestration drainage, increases the risk of peripheral organ damage and other complications (3). Computed tomography (CT) urography (CTU) multiphase enhanced scan can obtain the three-dimensional image of the entire urinary system including the calyces, renal pelvis, ureter and bladder (4). By combining with multi-planar reconstruction (MPR) post-processing technology, the location of the cyst and its relationship with the collection system can be comprehensively assessed (5). The aim of this study was to evaluate the safety and efficacy of MPR-CTU combined with intraoperative ultrasonography to guide ureteroscopy in the treatment of renal cystic disease.

\section{Materials and methods}

Object data. A total of 68 patients diagnosed with CDK charged into the First Affiliated Hospital of Huzhou Teacher's College from June 2014 to September 2016 was continuously selected, inclusion criteria: i) Recurrent or persistent lumbar pain, the conservative treatment was invalid; ii) repeated urinary tract infection or merged with calculus, poor effect after conservative treatment; iii) imaging examination suggested cysts endogenous growth, kidney collection system compression, maximum diameter $>4 \mathrm{~cm}$, normal renal function or mild to moderate damage iv) blood pressure and blood sugar can be controlled in the normal range; and v) complete clinical data, informed consent obtained. Exclusion criteria: i) Past kidney surgery history, combined with renal abscess and kidney tumors; ii) severe heart, liver, lung, brain and other organ dysfunction, intolerance to the risk of surgery and 
Table I. Baseline data of the two groups.

\begin{tabular}{|c|c|c|c|c|}
\hline Groups & Control, $(n=34)$ & Observation, $(n=34)$ & $t / \chi^{2}$ & P-value \\
\hline Male/Female & $20 / 14$ & $18 / 16$ & 0.239 & 0.625 \\
\hline Age (years) & $53.2 \pm 8.9$ & $54.6 \pm 11.2$ & 0.196 & 0.867 \\
\hline Unilateral/bilateral cyst & $22 / 12$ & $20 / 14$ & 0.249 & 0.618 \\
\hline Simple cysts/polycystic kidney & $25 / 9$ & $23 / 11$ & 0.283 & 0.595 \\
\hline Cyst position, n (\%) & & & 0.340 & 0.987 \\
\hline Renal pelvis & $16(47.1)$ & $18(52.9)$ & & \\
\hline Superior pole & $5(14.7)$ & $5(14.7)$ & & \\
\hline Medium pole & $5(14.7)$ & $4(11.8)$ & & \\
\hline Inferior pole & $3(8.8)$ & $3(8.8)$ & & \\
\hline Multiple calices & $5(14.7)$ & $4(11.8)$ & & \\
\hline Cyst diameter (cm) & $5.5 \pm 1.4$ & $5.6 \pm 1.5$ & 0.086 & 0.924 \\
\hline \multicolumn{5}{|l|}{ Combined disease, n (\%) } \\
\hline Kidney stones & $8(23.5)$ & $9(26.5)$ & 0.078 & 0.779 \\
\hline Localized calyceal water & $6(17.6)$ & $8(23.5)$ & 0.360 & 0.549 \\
\hline Hypertension & $10(29.4)$ & $6(17.6)$ & 1.308 & 0.253 \\
\hline Diabetes & $5(14.7)$ & $4(11.8)$ & 0.000 & 1.000 \\
\hline Coronary heart disease & $3(8.8)$ & $3(8.8)$ & 0.000 & 1.000 \\
\hline
\end{tabular}

anesthesia; and iii) deformities in urinary system development, ureteroscope cannot pass.

The patients were divided into control and observation group by random number method $(\mathrm{n}=34)$. The baseline data of the two groups were comparable (Table I).

The study was approved by the Ethics Committee of the First Affiliated Hospital of Huzhou Teacher's College and informed consents were signed by the patients and/or guardians.

Research methods. Two groups of patients both underwent ureteroscopic renal cysts incision and drainage, which was performed by the same surgical and nursing team according to the standard surgical procedure. The control group was under ultrasound guidance, the observation group also combined with MPR-CTU technology, the procedures were as follows: The location of collection system and cyst was observed under ureteroscope, cyst wall was blue and transparent and processed to the collection system, cyst wall was generally foggy after incision, the wall folds were diverticulum-like; the wall of hydronephrosis was smooth, the mucosa was pink, the capillaries were obvious; the wall was cut about $2.5-3.5 \mathrm{~cm}$ with $200 \mu \mathrm{m}$ holmium laser fiber, so that the cysts and the collection system were connected. Low energy $(0.8 \mathrm{~J})$ and low frequency $(10-15 \mathrm{~Hz})$ was used for thin wall cyst, low energy $(0.8 \mathrm{~J})$ and high frequency $(20-30 \mathrm{~Hz})$ was used for thick wall cyst, high energy (1.0-1.5 J) and low frequency $(10-15 \mathrm{~Hz})$ was used for hemostasis, holmium laser irradiation distance should not be too close or too far $(2 \mathrm{~mm})$.

The cut wall was burnt and coagulated, in order to avoid the hyperplasia and closure of the wall; holmium laser lithotripsy was used for combined ipsilateral kidney stones, double $\mathrm{J}$ tube was indwelled after operation, the proximal end was indwelled in the cysts for drainage, 1 month after the operation, reexamined by $\mathrm{CT}$ and double $\mathrm{J}$ tube was removed. CTU were scanned in the renal arterial phase, renal parenchyma and excretion phase. The original images were processed by computer and then three-dimensionally reconstructed. The stereo images with high special resolution were obtained. The position relationship between the endogenous renal cysts and the front and the rear of renal pelvis, the adjacency relation with the collection system was accurately identified (6). MPR was adjusted by transverse axial, coronal, sagittal position and multi-directionally: The marginal zone nearest to the calyx and the cyst was taken, the fenestration drainage site was decided when the calyx and the cyst was located the closest to the three axial planes by locating simultaneously in the transverse axis, coronal, sagittal position (7). The cursor line perpendicular to each other in the coronal, sagittal and axial images can be adjusted to show real-time position and plane images, providing multi-directional and multi-angle observation, a series of axial image was reorganized along the line, the reconstruction of the cyst on the coronal plane, sagittal plane or any slope can be done, providing the target calyx incision position safely and accurately (Figs. 1 and 2).

The observed indicators. The operative success rate and mean cystic treatment time, intraoperative and postoperative complications, the total effective rate after 1 month follow-up were compared between the two groups. Treatment effect was divided into cure, improved and ineffective, in which cure was complete disappearance of clinical symptoms and signs, no complications, no recurrence after 6 months of follow-up; improved was clinical symptoms and signs significantly alleviated, no obvious complications, no recurrence after 6 months of follow-up; invalid was surgical failure or no significant relief or deterioration for clinical symptoms and signs, or associated with serious complications. Total efficiency $=$ (cure + improved)/total number of cases $x 100 \%$.

Statistical analysis. Statistical analysis was performed using SPSS20.0 software (SPSS Inc., Chicago, IL, USA). 

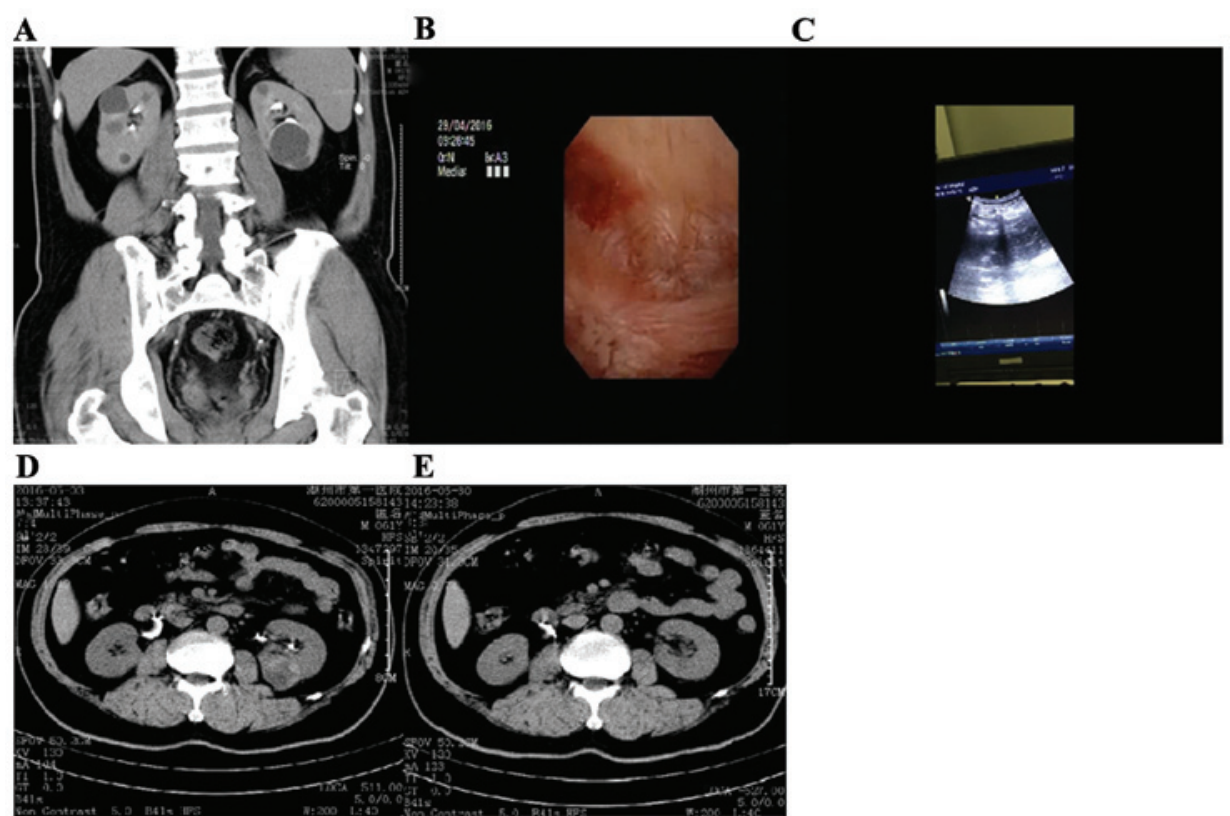

Figure 1. Control group using intraoperative ultrasound guidance. (A) Preoperative two-dimensional CT showed bilateral polycystic kidney; (B and C) intraoperative left ultrasound guidance; (D) 4 days after operation showed left side hematoma; (E) both sides returned to normal 1 month after operation. CT, computed tomography.

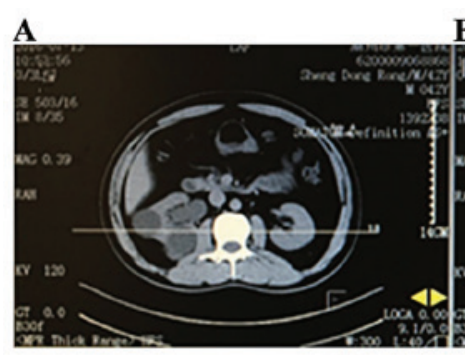

E B C

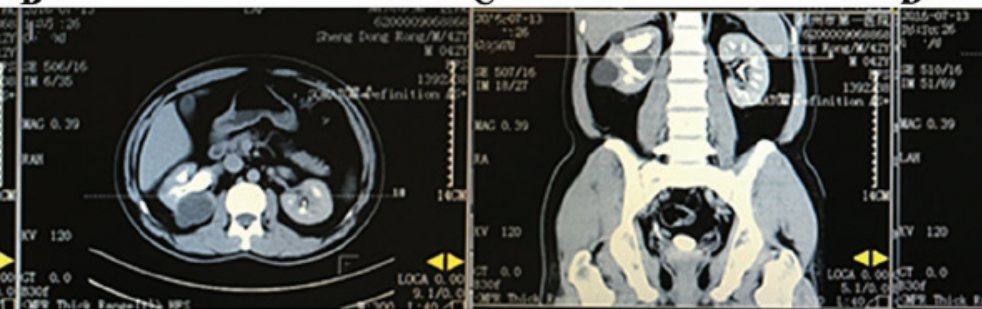

$\mathbf{F}$

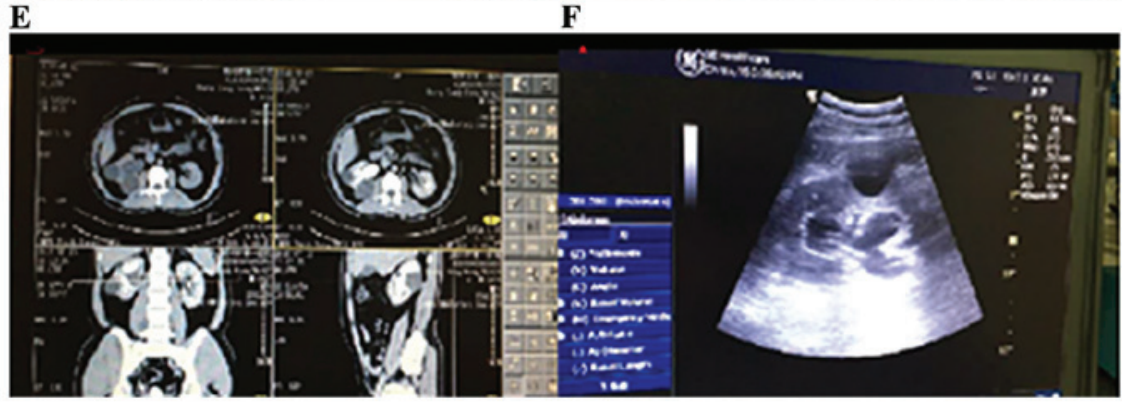

Figure 2. The observation group adopted MPR-CTU combined with intraoperative ultrasound guidance. (A-D) The preoperative CTU showing multiple renal cysts, renal pelvis cysts underwent ureteroscope resection, and intermediate cysts were not suitable for ureteroscope resection; (E) the parapelvis MPR-CTU; (F) intraoperative ultrasound shows the wall was too thick to find, located in the bottom of the superior calyx, parapelvis part was cut by ultrasound positioning, exogenous intermediate cyst cavity was thick from the collection system. MPR, multi-planar reconstruction, CT, computed tomography urography.

The measurement data were expressed as mean \pm standard deviation, and the independent samples t-test was used for comparison between groups. The enumeration data were expressed as the number of cases or (\%), comparison between groups using $\chi^{2}$ test; $\mathrm{A} \mathrm{P}<0.05$ was considered to indicate a statistically significant difference.

\section{Results}

The success rate of surgery and the average cyst treatment time. There was no significant difference in the operative success rate and cyst treatment time between the two groups (P>0.05) (Table II).

Intraoperative and postoperative complications. The incidence of intraoperative and postoperative complications was significantly lower in the observation group than in the control group, the difference was statistically significant $(\mathrm{P}<0.05)$. (Table III).

Follow-up effect. The total effective rate of the observation group was significantly higher than the control group, the difference was statistically significant $(\mathrm{P}<0.05)$ (Table IV). 
Table II. The success rate of surgery and the average cyst treatment time.

\begin{tabular}{lcccc}
\hline Groups & $\begin{array}{c}\text { Success, } \\
\mathrm{n}(\%)\end{array}$ & $\begin{array}{c}\text { Cyst not found, } \\
\mathrm{n}(\%)\end{array}$ & $\begin{array}{c}\text { Cyst excision difficult, } \\
\mathrm{n}(\%)\end{array}$ & $\begin{array}{c}\text { Average cyst treatment } \\
\text { time, (min) }\end{array}$ \\
\hline Control $(\mathrm{n}=34)$ & $28(82.4)$ & $3(8.8)$ & $3(8.8)$ & $36.7 \pm 12.5$ \\
Observation $(\mathrm{n}=34)$ & $30(88.2)$ & $1(2.9)$ & $3(8.8)$ & $32.2 \pm 10.3$ \\
$\mathrm{t} / \chi^{2}$ & 0.469 & & & 1.625 \\
P-value & 0.493 & & & 0.239 \\
\hline
\end{tabular}

Table III. Intraoperative and postoperative complications, n (\%).

\begin{tabular}{lcccccc}
\hline Groups & $\begin{array}{c}\text { Intracapsular } \\
\text { hematoma }\end{array}$ & Perforation & $\begin{array}{c}\text { Urinary } \\
\text { fistula }\end{array}$ & Infection & $\begin{array}{c}\text { Renal } \\
\text { failure }\end{array}$ & $\begin{array}{c}\text { Total } \\
\text { complications }\end{array}$ \\
\hline Control $(\mathrm{n}=34)$ & $3(8.8)$ & $3(8.8)$ & $2(5.9)$ & $1(2.9)$ & $1(2.9)$ & $10(29.4)$ \\
Observation $(\mathrm{n}=34)$ & $1(2.9)$ & $1(2.9)$ & $1(2.9)$ & 0 & 0 & $3(8.8)$ \\
$\chi^{2}$ & & & & & 4.660 \\
P-value & & & & & 0.031 \\
\hline
\end{tabular}

Table IV. Follow-up treatment effect, n (\%).

\begin{tabular}{lcccc}
\hline Groups & Cure & Improved & Invalid & $\begin{array}{c}\text { Total } \\
\text { efficiency }\end{array}$ \\
\hline $\begin{array}{l}\text { Control } \\
(\mathrm{n}=34)\end{array}$ & $10(29.4)$ & $10(9.4)$ & $14(41.2)$ & $20(58.8)$ \\
$\begin{array}{l}\text { Observation } \\
(\mathrm{n}=34)\end{array}$ & $19(55.9)$ & $9(26.5)$ & $6(17.6)$ & $28(82.4)$ \\
$\chi^{2}$ & & & & \\
P-value & & & & 4.533 \\
\hline
\end{tabular}

\section{Discussion}

The theory basis of renal cystic disease are: i) Polycystic kidney disease is occult, with long duration, a large number of cysts increase chronically and progressively causing renal parenchyma squeezed and degenerated, gradually replaced by fibrous tissue, renal function is gradually diminished, ultimately progress to chronic renal failure (8); ii) due to decreased renal filtration rate, water and sodium retention, renal cortex ischemia, renin - aldosterone system is activated, leading to secondary hypertension, further exacerbate renal function deterioration (9); iii) with the cysts increasing, the obstruction factors inside and outside the cavity cause the oppression of functional nephrons and blood vessels, resulting in renal ischemic damage and functional renal unit reduction; iv) polycystic kidney capsule has epidermal growth factor, cytokines and other factors, which can promote renal epithelial cell proliferation, change the renal interstitial, playing an important role in the formation and development of cysts (10).

Ultrasound guided percutaneous drainage is the most common treatment of renal cystic disease, with minimum surgical trauma; but the cyst disappearance rate is $<50 \%$, the recurrence rate is as high as 17 to $44 \%$ (11); especially not ideal for cyst with diameter $>10 \mathrm{~cm}$; it is restricted by the location of the cyst, with difficulties of puncture on the cyst located at the renal ventral side and the superior pole (12); for the cyst close to the collection system, sclerosing agent may penetrate into the collection system, causing serious complications (13); obvious pain may occur after the injection of cysts; renal-derived cyst, localized hydronephrosis are contraindications; it cannot handle kidney stones, the malignant transformation is difficult to be identified (14). Laparoscopic renal cyst decompression has significantly reduced recurrence rate, the surgical effect is better than ultrasound-guided puncture, and is similar to open surgery (15); drawbacks are traumatic bleeding and other complications; difficult to operate on parapelvic cyst, endogenous cyst (16); has high incidence of postoperative urinary fistula after localized hydronephrosis, poor repeatability and often needs re-operation (17). Internal drainage combined with decompression can make the larger cysts combined and connected to the collection system, and perform decompression to deeper cysts or connect them to larger cysts, then communicate with the connection system, therefore most of the cyst fluid is excreted from the urinary tract, the drainage is more reasonable and smooth, which is confirmed by a number of studies $(18,19)$ has better clinical safety and effectiveness. It is also concluded from this study that there is no significant difference between the two groups in terms of the incidence of surgery and operation time on cysts, the incidence of operative and postoperative complications in the observation group was significantly lower than that in the control group. After 1-month follow-up, the total effective rate of the observation group was significantly higher than that of the control group. The three-dimensional image of the entire urinary system, including the calyx, renal pelvis, ureter and bladder can be obtained through CT urography (CTU) multi-phase dynamic enhanced scan. By combining with MPR image post-processing technique, the location of cysts 
and their relationship with the collection system can be evaluated comprehensively. Therefore, we believe that MPR-CTU combined with renal cyst incision and drainage under intraoperative ultrasound guided ureteroscope has a higher safety and effectiveness in the treatment of renal cystic disease, it is worthy of clinical application.

Studies found that $(20,21)$ when treating endogenous cyst with flexible ureteroscope, the best incision position cannot be precisely determined; it was difficult to find the best laser rupture position for the cyst in the calyx and pelvis by ureteroscope; the incision cannot be found because of the thick cyst wall. Therefore, effective assessment of whether the flexible ureteroscope can handle specific endogenous cysts before surgery and how to effectively locate the cyst is a key factor for the success of surgery. The main innovative points of this study are: flexible ureteroscope technology is currently the most minimally invasive technique, treating the cyst via the urinary approach is most in line with the natural cavity path, with characteristics of repeatable operating, suitable for recurrence patients after open or laparoscopic surgery. There is no clinical report of MPR-CTU assisted ultrasonography in the treatment of renal cystic disease with ureteroscope localization within renal calyx. The deficiency of this research is the small sample size, short follow-up time, it still needs further verification.

\section{Acknowledgements}

The present study was supported by the Public Welfare Marjor Project of the Bureau of Science \& Technology (no. 2014GZB03).

\section{References}

1. Mao X, Xu G, Wu H and Xiao J: Ureteroscopic management of asymptomatic and symptomatic simple parapelvic renal cysts. BMC Urol 15: 48, 2015.

2. Xu L, Rong Y, Wang W, Lian H, Gan W, Yan X, Li X and Guo H: Percutaneous radiofrequency ablation with contrast-enhanced ultrasonography for solitary and sporadic renal cell carcinoma in patients with autosomal dominant polycystic kidney disease. World J Surg Oncol 14: 193, 2016.

3. Rahbari-Oskoui F, Mittal A, Mittal P and Chapman A: Renal relevant radiology: Radiologic imaging in autosomal dominant polycystic kidney disease. Clin J Am Soc Nephrol 9: 406-415, 2014.

4. Ramanathan S, Kumar D, Khanna M, Al Heidous M, Sheikh A, Virmani V and Palaniappan Y: Multi-modality imaging review of congenital abnormalities of kidney and upper urinary tract. World J Radiol 8: 132-141, 2016.

5. Chapman $\mathrm{AB}$ and Wei W: Imaging approaches to patients with polycystic kidney disease. Semin Nephrol 31: 237-244, 2011.

6. Pallwein-Prettner L, Flöry D, Rotter CR, Pogner K, Syré G, Fellner C, Frauscher F, Aigner F, Krause FS and Fellner F: Assessment and characterisation of common renal masses with CT and MRI. Insights Imaging 2: 543-556, 2011.
7. Rule AD, Sasiwimonphan K, Lieske JC, Keddis MT, Torres VE and Vrtiska TJ: Characteristics of renal cystic and solid lesions based on contrast-enhanced computed tomography of potential kidney donors. Am J Kidney Dis 59: 611-618, 2012.

8. Ta MH, Rao P, Korgaonkar M, Foster SF, Peduto A, Harris DC and Rangan GK: Pyrrolidine dithiocarbamate reduces the progression of total kidney volume and cyst enlargement in experimental polycystic kidney disease. Physiol Rep 2: e12196, 2014.

9. Dell KM: The spectrum of polycystic kidney disease in children. Adv Chronic Kidney Dis 18: 339-347, 2011.

10. Saigusa T and Bell PD: Molecular pathways and therapies in autosomal-dominant polycystic kidney disease. Physiology (Bethesda) 30: 195-207, 2015.

11. Ali TA,Abdelaal MA,Enite A and Badran YA: Ultrasound-guided percutaneous sclerotherapy of simple renal cysts with n-butyl cyanoacrylate and iodized oil mixture as an outpatient procedure. Urol Ann 8: 51-55, 2016.

12. Akoh JA: Current management of autosomal dominant polycystic kidney disease. World J Nephrol 4: 468-479, 2015.

13. Yu JH, Du Y, Li Y, Yang HF, Xu XX and Zheng HJ: CT-guided sclerotherapy for simple renal cysts: Value of ethanol concentration monitoring. Korean J Radiol 15: 80-86, 2014.

14. Ars E, Bernis C, Fraga G, Martínez V, Martins J, Ortiz A, Rodríguez-Pérez JC, Sans L and Torra R: Spanish working group on inherited kidney disease: Spanish guidelines for the management of autosomal dominant polycystic kidney disease. Nephrol Dial Transplant 29: 95-105, 2014.

15. Agarwal M, Agrawal MS, Mittal R and Sachan V: A randomized study of aspiration and sclerotherapy versus laparoscopic deroofing in management of symptomatic simple renal cysts. J Endourol 26: 561-565, 2012.

16. Riella C, Czarnecki PG and Steinman TI: Therapeutic advances in the treatment of polycystic kidney disease. Nephron Clin Pract 128: 297-302, 2014.

17. Millar M, Tanagho YS, Haseebuddin M, Clayman RV, Bhayani SB and Figenshau RS: Surgical cyst decortication in autosomal dominant polycystic kidney disease. J Endourol 27: 528-534, 2013.

18. Papatsoris AG, Kachrilas S, Howairis ME, Masood $\mathbf{J}$ and Buchholz N: Novel technologies in flexible ureterorenoscopy. Arab J Urol 9: 41-46, 2011.

19. Luo Q, Zhang X, Chen H, Liu Z, Chen X, Dai Y and Zhao Z: Treatment of renal parapelvic cysts with a flexible ureteroscope. Int Urol Nephrol 46: 1903-1908, 2014

20. Zhao Q, Huang S, Li Q, Xu L, Wei X, Huang S, Li S and Liu Z: Treatment of parapelvic cyst by internal drainage technology using ureteroscope and Holmium laser. West Indian Med J 64: 230-235, 2015.

21. Basiri A, Hosseini SR, Tousi VN and Sichani MM: Ureteroscopic management of symptomatic, simple parapelvic renal cyst. J Endourol 24: 537-540, 2010.

(7) $\Theta$ This work is licensed under a Creative Commons Attribution-NonCommercial-NoDerivatives 4.0 International (CC BY-NC-ND 4.0) License. 